\section{Dealing with poisoned patients}

The Poisoned Patient: The Role of the Laboratory. (Ciba Foundation Symposium 26, New Series.) Pp. viii +325. (Associated Scientific: Amsterdam, Oxford and New York, 1974.) Dfl. 55; $\$ 21.20$.

This volume comprises 17 papers devoted to various aspects of poisoning; each is accompanied by a very full and frequently thought-provoking discussion.

Almost one third of the papers deal with drug detection and assay techniques, such as gas chromatography linked with mass spectometry, radio immunoassay and associated methodological problems. The controversy over the place of the laboratory in the management of patients who have taken overdoses is covered by Newton and Prescott of Edinburgh who argue spiritedly against Goulding of London and the North American contingent. My impressions are that all of the participants advocate conservative management of the majority of patients and that differences in emphasis of active treatment and demands for drug profile results would disappear if all specialists saw a similar spectrum of patients.

The least well covered aspect in the symposium concerns the role of the laboratory in detecting and preventing drug induced disease. Leach's paper on this subject is mainly concerned with refinement in existing drug assay techniques. The tantalising problem of converting present methods which are mainly concerned with single 'once off' assays into systems capable of dealing with many samples is barely touched on, and no mention is made of the claims of improvement in patient management that have followed the introduction of procedures such as the immunoassay of digoxin. It is estimated that between 5 and $10 \%$ of hospital beds are filled by patients suffering from drug-induced diseases. Presumably, that figure indicates the tip of the iceberg with respect to the total quantity of drug-induced ill health.

Dole contributes a stimulating paper on the role of the laboratory in the treatment of narcotic poisoning. In the US it is a federal requirement that the urine of those patients receiving maintenance methadone be tested weekly. That, however, has been found to be positively detrimental to the rehabilitation of patients, as it fosters noncooperative attitudes.

Repeatedly during the discussions it is claimed that doctors are not sufficiently trained in clinical pharma- cology, so that no matter how sophisticated and accurate are the assay techniques used, the data obtained will be wasted. This book should stimulate interest in clinicians, thus going some way towards overcoming this very real gap in medical education and helping towards the establishment of useful collaboration between clinical and laboratory workers. Noel Wright

\section{Biological interface}

Biological Interfaces: An Introduction to the Surface and Colloid Science of Biochemical and Biological Systems. By Malcolm N. Jones. Pp. ix +240 . (Elsevier Scientific: Amsterdam, Oxford and New York, 1975.) Dfl.34; $\$ 13.25$.

THERE is no question that some knowledge of surface and colloid chemistry can be a valuable aid to the understanding of a range of biological problems. To date, however, there have been no serious attempts to present in a systematic manner those aspects of surface chemistry which are particularly relevant to biology. Dr Jones has undertaken this quite difficult task and, though he has not produced a definitive work on the subject, he has certainly produced a very readable little volume which should be useful to everyone from undergraduates upwards.

There are eight chapters on topics including surface and interfacial tension, monolayers, micelles, proteinsurfactant interactions, electrical double layers, cell surfaces and cell contacts and artificial membranes. In each instance the surface chemical principles are discussed with special regard for biological systems and numerous references are given to the original literature. Herein lies the first of my criticisms, that is, that the references are not, in some cases, as up-todate as they might be. It may be that this is difficult to avoid in a book on a relatively fast-moving field but, even so, this point should be appreciated. My second concern is that in one or two instances I should like to have seen a more critical presentation of the material. For example, it is not reassuring to find the author putting in a good word for the Pauling-Miller anaesthesia hypothesis, or to see Clements' theory of alveolar stability presented as established fact, or to find that the evidence on the water permeability of bilayers has been inadequately summed up. On most topics, however, the discussion and assessment of the present situation are refreshingly good and, for those starting out in the field, this is a book well worth reading.

D. A. Haydon
The Flavonoids

Edited by J. B. HARBORNE, T. J. MABRY and HELGA MABRY

July 1975: 1162 pages: 41211960 9: hardback: f27.50

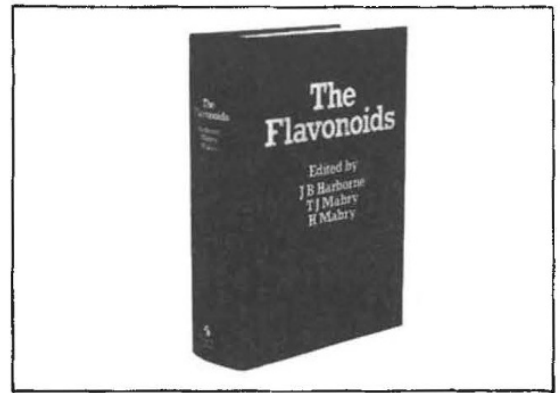

This book provides a comprehensive and definitive account of the flavonoids, one of the most important groups of plant substances. The text covers their recent chemistry, biochemistry, natural distribution and biological importance. The first few chapters deal with techniques of isolation and spectral measurements and with chemical synthesis. There then follow individual chapters covering the fifteen known classes of flavonoid. A significant feature of these chapters is the comprehensive and up-to-date tables listing all known structures within each class of compound. The remaining chapters deal with the enzymology, biosynthesis, metabolism. physiology, systematic distribution, evolution and function of the flavonoids.

\section{Rheometry}

\section{K. WALTERS}

July 1975: 288 pages: illustrated: 41212090 9: hardback: $£ 10.00$

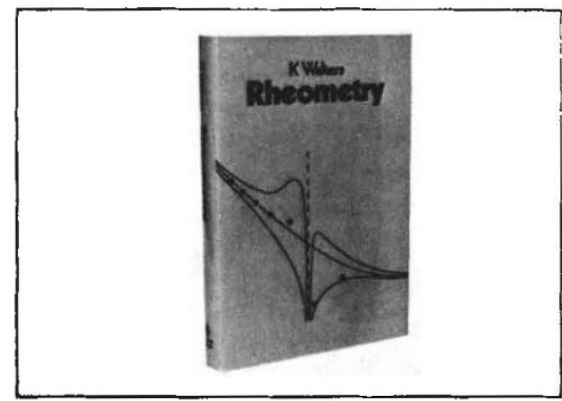

There are now many commercially available rheometers with varying degrees of sophistication. The present book is intended to be a text book for such instruments as well as providing a background to rheometry in general.

The development in the book is based on the proposition that there are two basic objectives in rheometry. The first involves a straightforward attempt to characterize the behaviour of nonNewtonian liquids in a number of simple (rheometrical) flow situations, with a view to correlating material behaviour with either molecular structure or observed behaviour in practical situations. The second objective concerns the construction of rheological equations of state for the liquids which can be later used in the solution of flow problems of practical importance.

The author is a mathematician but a genuine attempt has been made to avoid unnecessary mathematical rigour.

\section{Chapman \& Hall}

11 New Fetter Lane, London EC4P 4EE 